\title{
Kolmogorov-Sinai entropy for dilute gases in equilibrium
}

\author{
H. van Beijeren, ${ }^{1}$ J. R. Dorfman, ${ }^{2}$ H. A. Posch, ${ }^{3}$ and Ch. Dellago ${ }^{3,4}$ \\ ${ }^{1}$ Institute for Theoretical Physics, University of Utrecht, Postbus 80006, 3508 TA Utrecht, The Netherlands \\ ${ }^{2}$ Institute for Physical Science and Technology and Department of Physics, University of Maryland, College Park, Maryland 20742 \\ ${ }^{3}$ Institut für Experimentalphysik, Universität Wien, Boltzmanngasse 5, A-1090 Wien, Austria \\ ${ }^{4}$ Department of Chemistry, University of California at Berkeley, Berkeley, California 94720
}

(Received 18 June 1997)

\begin{abstract}
We consider the density expansion of the Kolmogorov-Sinai (KS) entropy per particle for a dilute gas in equilibrium, and use methods from the kinetic theory of gases to compute the leading term. For an equilibrium system, the KS entropy $h_{\mathrm{KS}}$ is the sum of all of the positive Lyapunov exponents characterizing the chaotic behavior of the gas. We compute $h_{\mathrm{KS}} / N$, where $N$ is the number of particles in the gas. This quantity has a density expansion of the form $h_{\mathrm{KS}} / N=a \nu[-\ln \tilde{n}+b+O(\tilde{n})]$, where $\nu$ is the single-particle collision frequency and $\tilde{n}$ is the reduced number density of the gas. The theoretical values for the coefficients $a$ and $b$ are compared with the results of computer simulations, with excellent agreement for $a$, and less than satisfactory agreement for $b$. Possible reasons for this difference in $b$ are discussed. [S1063-651X(97)06911-0]
\end{abstract}

PACS number(s): 05.45.+b, 05.20.Dd

One of the important quantities characterizing the chaotic behavior of a dynamical system is the Kolmogorov-Sinai $(\mathrm{KS})$ entropy, $h_{\mathrm{KS}}[1,2]$. If the system is isolated and closed, i.e., there is no escape of particles from the system, then, according to Pesin's theorem [2], $h_{\mathrm{KS}}$ is the sum of all the positive Lyapunov exponents of the system, where the Lyapunov exponents characterize the rate of exponential separation of the system's trajectories in phase space. This quantity is of considerable interest because a positive KS entropy implies that the system is chaotic with strong mixing and ergodic properties, and because the KS entropy measures the rate at which information about the phase space trajectories is produced by the dynamics. Further, sums of Lyapunov exponents figure prominently in the expressions for transport coefficients of fluids in terms of dynamical quantities $[3,4]$. Recently, methods based upon the kinetic theory of gases have been applied to compute the chaotic properties of simple systems such as hard disk and hard sphere Lorentz gases [5-8], as well as Lorentz lattice gases [9,10]. This work has shown that it is possible to calculate theoretically Lyapunov exponents for Lorentz gases at low density under a variety of equilibrium and nonequilibrium situations. The theoretical results agree very well with computer simulations for the same quantities. Here we show how kinetic theory methods can be extended to a calculation of the leading term in the density expansion of the equilibrium KS entropy of dilute gases composed of $N$ particles interacting with shortrange forces. This extension of the methods developed earlier for Lorentz gases to systems of $N$ moving particles allows us to begin a thorough exploration of the chaotic properties of systems with large numbers of moving particles, as well as of Lorentz gases, both in and outside equilibrium states. In subsequent publications van Beijeren and van Zon [11] will describe methods to obtain the largest positive Lyapunov exponent for a dilute gas in equilibrium.

We consider a gas composed of $N$ identical particles in equilibrium. The particles obey classical mechanics, each have mass $m=1$, and their positions and velocities are de- noted by $\vec{r}_{i}$ and $\vec{v}_{i}$, respectively, with $i=1, \ldots, N$. The particles interact with strongly repulsive, short-range forces with a finite range of the interparticle force, $\sigma$. In the case that the particles are hard disks or hard spheres, $\sigma$ is the diameter of each particle. Our goal is to determine the KS entropy per particle, $h_{\mathrm{KS}} / N$, in the thermodynamic limit, assuming that the system is in thermal equilibrium at temperature $T$, and at low densities with $n \sigma^{d} \ll 1$, where $n$ is the number density, $n=N / V, V$ is the volume of the system, and $d$ is the dimension of the system, $d=2$ and 3 .

We consider the trajectory of our system on the constant energy surface in the $2 d N$-dimensional phase space, $\Gamma$, starting from some initial point $\mathbf{X}_{0}=\left(\vec{r}_{1}(0), \vec{v}_{1}(0), \vec{r}_{2}(0)\right.$, $\left.\vec{v}_{2}(0), \ldots, \vec{r}_{N}(0), \vec{v}_{N}(0)\right)$. We then consider a bundle of trajectories that start at infinitesimally nearby points in phase space, with each trajectory in the bundle denoted by $\mathbf{X}(0)$ $+\delta \mathbf{X}(0)$, for some infinitesimal $\delta \mathbf{X}(0)=\left(\delta \vec{r}_{1}(0)\right.$, $\left.\delta \vec{v}_{1}(0), \ldots, \delta \vec{r}_{N}(0), \delta \vec{v}_{N}(0)\right)$. The time rate of separation of this trajectory bundle in the various directions perpendicular to the direction of flow, if exponential, is characterized by a set of nonzero Lyapunov exponents which are positive in the expanding directions and negative in the contracting ones. The KS entropy is, for our system, given by the sum of all of the positive Lyapunov exponents, by Pesin's theorem $[1,2]$. In order to determine the Lyapunov exponents and their sum, we need to follow the dynamics of the trajectory bundle over a time $t$ very large compared to some characteristic microscopic time $t_{0}$, which for low-density systems can be taken to be the mean free time between collisions for a typical particle. Recently Dellago, Posch and Hoover, as well as Gaspard and Dorfman, obtained the equations of motion for a trajectory bundle in phase space for a system of hard disk or hard sphere particles $[4,12,13]$. These equations also apply to any system of particles with strong short-range interaction potentials, provided we neglect effects due to collisions involving more than two particles at a time, or due to the finite duration of collisions taking place in the fluid. To lowest 
order in the density, these multiple collision and finite collision time effects may be ignored, and the dynamics may be thought of as periods of free motion of the particles punctuated by binary collisions between pairs of particles. During the free motion, the positions and velocities of the particles change with time according to

$$
\begin{gathered}
\vec{r}_{i}(t)=\vec{r}_{i}(0)+\vec{v}_{i}(0) t, \\
\vec{v}_{i}(t)=\vec{v}_{i}(0) \quad \text { for } i=1, \ldots, N .
\end{gathered}
$$

Here the time $t=0$ represents the instant of the last binary collision in the system, and time $t$ is some time between the last binary collision and the next. When a collision takes place between particles $k$ and $l$, say, there is an effectively instantaneous change in the velocities of particles $k$ and $l$ while the positions and velocities of all of the other $N-2$ particles are unaffected. Immediately after the $k-l$ collision, the positions and velocities of the two particles, $k$ and $l$ are given by

$$
\begin{gathered}
\vec{v}_{k}^{\prime}=\vec{v}_{k}+\left[\vec{v}_{l k} \cdot \hat{n}\right] \hat{n}, \\
\vec{v}_{l}^{\prime}=\vec{v}_{l}-\left[\vec{v}_{l k} \cdot \hat{n}\right] \hat{n}, \\
\vec{r}_{l}=\vec{r}_{k}+\sigma \hat{n} .
\end{gathered}
$$

Here $\hat{n}$ is a unit vector from the center of particle $k$ to the center of particle $l$ at the point of closest approach during the binary collision, $\vec{v}_{l k}=\vec{v}_{l}-\vec{v}_{k}$ and $\vec{v}_{l k} \cdot \hat{n} \leqslant 0$. The free motion of the other $N-2$ particles $i \neq k$ and $l$ is not affected by the $k-l$ collision. Now we consider the other trajectories in the nearby bundle. We assume that the bundle is sufficiently narrow that all trajectories in the bundle exhibit the same collisions in the same sequence, with slight differences in the times of the collisions, in the velocities and positions before and after the collisions, and in the points of closest approach. The analysis of Dellago, Posch, and Hoover leads immediately to equations of motion for the deviations of the positions and velocities in the trajectory bundle from the main trajectory. During the free motion between collisions, $\delta \vec{r}_{i}$ and $\delta \vec{v}_{i}$ evolve according to

$$
\begin{gathered}
\delta \vec{r}_{i}(t)=\delta \vec{r}_{i}(0)+t \delta \vec{v}_{i}(0), \\
\delta \vec{v}_{i}(t)=\delta \vec{v}_{i}(0) .
\end{gathered}
$$

Whenever a collision takes place in the system, the deviations in positions and velocities of the colliding particles are directly affected, while those of the other particles are not. The velocity deviations for the colliding pair, say particles $k$ and $l$, can be obtained from Eqs. (3) and (4) by taking linear deviations; that is,

$$
\begin{gathered}
\delta \vec{V}_{l k}^{\prime} \equiv \frac{1}{2}\left[\delta \vec{v}_{k}^{\prime}+\delta \vec{v}_{l}^{\prime}\right]=\delta \vec{V}_{l k}, \\
\delta \vec{v}_{l k}^{\prime} \equiv \delta \vec{v}_{l}^{\prime}-\delta \vec{v}_{k}^{\prime}=\delta \vec{v}_{l k}-2\left(\delta \vec{v}_{l k} \cdot \hat{n}\right) \hat{n} \\
-2\left[\left(\vec{v}_{l k} \cdot \delta \hat{n}\right) \hat{n}+\left(\vec{v}_{l k} \cdot \hat{n}\right) \delta \hat{n}\right],
\end{gathered}
$$

where, for later convenience, we introduce the center-ofmass velocity $\vec{V}_{l k}=\frac{1}{2}\left[\vec{v}_{k}+\vec{v}_{l}\right]$, in addition to the relative velocity of the colliding pair of particles. Here $\delta \hat{n}$ is the infinitesimal displacement of the unit vector in the direction of closest approach, due to the displacement of the trajectory, and $\hat{n} \cdot \delta \hat{n}=0$. To calculate $\delta \hat{n}$ we use the coupled equations for the location of the collision for both the undisplaced and the displaced trajectories:

$$
\begin{gathered}
\sigma \hat{n}=\vec{r}_{l, 0}+\tau_{l} \vec{v}_{l}-\vec{r}_{k, 0}-\tau_{k} \vec{v}_{k}, \\
\sigma \delta \hat{n}=\delta \vec{r}_{l, 0}-\delta \vec{r}_{k, 0}+\vec{v}_{l k} \delta \tau_{l k}+\tau_{l} \delta \vec{v}_{l}-\tau_{k} \delta \vec{v}_{k},
\end{gathered}
$$

where we have kept terms to linear order in the deviations. Here $\vec{r}_{l, 0}$ is the position of particle $l$ at the instant of its last collision with another particle in the system, and similarly for $\vec{r}_{k, 0}$. Also, $\tau_{l}$ is the time elapsed from the last collision of particle $l$ until its collision with $k$, with a similar definition for $\tau_{k}$. Finally $\delta \tau_{l k}$ is the difference in time between the $l-k$ collision for the displaced and the undisplaced trajectories. We assume here that in our calculation of $h_{\mathrm{KS}} / N$, the dominant terms will come from terms proportional to $T_{k l}=\left(\tau_{k}\right.$ $\left.+\tau_{l}\right) / 2$ which is on the order of the free time between collisions of particles in the system, and which scales inversely with the density of the gas. For low densities this free time can be quite large, and our assumption is that we can neglect the term $\delta \vec{r}_{l, 0}-\delta \vec{r}_{k, 0}$ in Eq. (11) compared to terms proportional to the times between collisions for each of the particles. This assumption will be discussed further in the concluding remarks. Then using the condition $\hat{n} \cdot \delta \hat{n}=0$, and changing to center-of-mass and relative velocities, we finally obtain equations for the change in the displaced center of mass and displaced relative velocities of particles $k$ and $l$ at the $k-l$ collision:

$$
\begin{gathered}
\delta \vec{v}_{i}^{\prime}=\delta \vec{v}_{i} \text { for } i \neq k, l \\
\delta \vec{V}_{k l}^{\prime}=\delta \vec{V}_{k l} \\
\delta \vec{v}_{l k}^{\prime}=\delta \vec{v}_{l k}-2\left(\delta \vec{v}_{l k} \cdot \hat{n}\right) \hat{n}-\left(\frac{2}{\sigma}\right)\left\{t _ { l k } \left[\left(\left(\vec{v}_{l k} \cdot \delta \vec{V}_{k l}\right)\right.\right.\right. \\
\left.\left.-\frac{v_{l k}^{2}}{\left(\vec{v}_{l k} \cdot \hat{n}\right)}\left(\delta \vec{V}_{k l} \cdot \hat{n}\right)\right) \hat{n}+\delta \vec{V}_{k l}\left(\vec{v}_{l k} \cdot \hat{n}\right)-\vec{v}_{l k}\left(\delta \vec{V}_{k l} \cdot \hat{n}\right)\right] \\
+T_{k l}\left[\left(\left(\vec{v}_{l k} \cdot \delta \vec{v}_{l k}\right)-\frac{v_{l k}^{2}}{\left(\vec{v}_{l k} \cdot \hat{n}\right)}\left(\delta \vec{v}_{l k} \cdot \hat{n}\right)\right) \hat{n}\right. \\
\left.\left.+\left(\vec{v}_{l k} \cdot \hat{n}\right) \delta \vec{v}_{l k}-\vec{v}_{l k}\left(\delta \vec{v}_{l k} \cdot \hat{n}\right)\right]\right\}
\end{gathered}
$$

Here $t_{l k}=\tau_{l}-\tau_{k}$. Using these equations we can express the change of the velocity deviations of all of the particles at a $k-l$ collision as a matrix $\mathbf{M}_{k l}$ acting on the column vector $\delta \mathbf{v}$ whose elements are the velocity deviations, $\delta \vec{v}_{i}$ for $i$ $=1, \ldots, N$, as $\delta \mathbf{v}^{\prime}=\mathbf{M}_{k l} \cdot \delta \mathbf{v}$. Here $\mathbf{M}_{k l}$ has the value 1 along the diagonal for the elements corresponding to the $N$ -2 particles which are not involved in the $k-l$ collision, and 
nonzero elements for the changes in the velocity deviations for particles $k$ and $l$, which can be obtained from Eqs. (13) and (14) in terms of the pre-collision velocity deviations and the times $T_{k l}$ and $t_{l k}$. All other matrix elements of $\mathbf{M}_{k l}$ are zero.

Thus by neglecting all of the deviations in positions at the instant of binary collisions $\delta \vec{r}_{i, 0}$, we can easily write an evolution equation for the velocity deviation vector as the result of a sequence of binary collisions in the system as

$$
\delta \mathbf{v}(t)=\mathbf{M}_{\alpha_{1}} \cdot \mathbf{M}_{\alpha_{2}} \cdots \mathbf{M}_{\alpha_{K(t)}} \cdot \delta \mathbf{v}(0) .
$$

In Eq. (15), we use the facts that the dynamics of the system is a sequence of binary collisions, labeled by the subscripts $\alpha_{j}$, with $\alpha_{K(t)}$ the last collision up to time $t$, and where only the velocities of two particles change, separated by freeparticle motions where none of the velocities change. For low densities, the product of matrices on the right-hand side of Eq. (15) can be thought of as a product of random $d N$ $\times d N$ matrices, since there are no correlations between the collisions in the sequences. The random elements of the sequence are the particles involved in the individual collisions, the collision parameters of each collision, and the time intervals, for each particle, between the collisions that it suffers. Under these circumstances all of the Lyapunov exponents and the KS entropy of the system can be obtained, in principle, by determining the eigenvalues of this product of random matrices, using the known distributions of free times and collision parameters for a dilute gas in equilibrium. The positive Lyapunov exponents and the KS entropy can be obtained by using the fact that almost all trajectories in phase space will separate with time $t$, as $t$ grows large, since the probability of finding two nearby, but otherwise randomly selected, trajectories that approach each other for arbitrarily long times, is vanishingly small. The negative Lyapunov exponents are obtained by considering the time reversed motion and using the fact that trajectories that approach each other in the forward time direction will separate in the time reversed motion. This analysis was applied to the random, dilute, three-dimensional Lorentz gas by Latz, van Beijeren, and Dorfman [7], who were able to calculate the positive and negative Lyapunov exponents by analyzing the products of random matrices similar to those in Eq. (15) along these lines. Due to the increased size of the matrices considered here, the determination of the individual Lyapunov exponents is still a formidable analytical problem. However the determination of the KS entropy is relatively elementary, as we now show.

Suppose that the eigenvalues of the product of the matrices on the right-hand side of Eq. (15) have the form $\exp \left(t \lambda_{i}\right)$. Then the logarithm of the determinant of this matrix product will have the form $t \Sigma \lambda_{i}$. Since almost all trajectories will lead to separation, only the positive exponents will appear in this sum. Further, if we assume that the system is ergodic then for long times $t$, all possible collision parameters and free times will appear in the matrices. Thus we can write

$$
h_{\mathrm{KS}}=\lim _{t \rightarrow \infty} \frac{1}{t} \sum_{j=1}^{j=K(t)} \ln \left|\operatorname{det} \mathbf{M}_{\alpha_{j}}\right|=\frac{N \nu}{2}\left\langle\ln \left|\operatorname{det} \mathbf{M}_{1,2}\right|\right\rangle,
$$

where $\nu$ is the average collision frequency per particle, so that the number of collisions taking place in the gas per unit time is $N \nu / 2$ (each collision involves two particles), and the angular brackets denote averages over the collision rates and parameters, the free time distributions, and the velocity distributions for a dilute gas in equilibrium. We have also used the indices 1 and 2 to label the colliding particles in a typical matrix M. This matrix has a particularly simple structure when expressed in terms of the deviations of the center-ofmass and relative velocities of particles 1 and 2 and the velocity deviations of particles $3, \ldots, N$, which are not affected by the 1-2 collision,

$$
\mathbf{M}_{1,2}=\left(\begin{array}{ccc}
\mathbf{1} & \mathbf{0} & \mathbf{0} \\
\mathbf{A} & \mathbf{B} & \mathbf{0} \\
\mathbf{0} & \mathbf{0} & \mathbf{1}
\end{array}\right) .
$$

Here we have organized the velocity deviation vector so that $\delta \mathbf{v}^{T}=\left(\delta \vec{V}_{12}, \delta \vec{v}_{21}, \delta \vec{v}_{3}, \ldots, \delta \vec{v}_{N}\right)^{T}$, where $T$ denotes a transpose. The four upper-left submatrices are all $d \times d$ dimensional. The $d \times d$ dimensional submatrices $\mathbf{A}$ and $\mathbf{B}$ are easily obtained from Eqs. (13) and (14) above. One easily sees that $\operatorname{det} \mathbf{M}_{1,2}=\operatorname{det} \mathbf{B}$, which is simple to calculate. Therefore we find that

$$
\begin{aligned}
h_{\mathrm{KS}} / N & =\frac{\nu}{2}\left\langle\ln \left[2 T_{12}\left|\vec{v}_{21}\right| /(\sigma \cos \theta)\right]\right\rangle \quad \text { for } d=2 \\
& =\nu\left\langle\ln \left|\left[2 T_{12}\left|\vec{v}_{21}\right| / \sigma\right]\right|\right\rangle \quad \text { for } d=3 .
\end{aligned}
$$

Here $\theta$ is the angle of incidence in a binary collision, with $-\pi / 2 \leqslant \theta \leqslant \pi / 2$ for two dimensions and $0 \leqslant \theta \leqslant \pi / 2$ in three dimensions. Equations (18) and (19) are the central theoretical results of this work. We have obtained explicit formulas for the KS entropy for a dilute gas of particles with strong short-range forces. We now give the explicit form of the integrals required for the calculation of $h_{\mathrm{KS}}$ for $d=2$ and 3 . For $d=2$ we obtain

$$
\begin{aligned}
h_{\mathrm{KS}} / N= & \frac{\nu}{2 \pi J_{2}} \int d \vec{v}_{1} \phi_{0}\left(\vec{v}_{1}\right) \int d \vec{v}_{2} \phi_{0}\left(\vec{v}_{2}\right)\left|\vec{v}_{12}\right| \\
& \times \int_{-\pi / 2}^{\pi / 2} d \theta \sigma(\pi-2 \theta) / \sigma \nu\left(v_{1}\right) \nu\left(v_{2}\right) \\
& \times \int_{0}^{\infty} d \tau_{1} \int_{0}^{\infty} d \tau_{2} e^{-\left[\tau_{1} \nu\left(v_{1}\right)+\tau_{2} \nu\left(v_{2}\right)\right]} \\
& \times \ln \left[2 T_{12}\left|\vec{v}_{12}\right| /(\sigma \cos \theta)\right] .
\end{aligned}
$$

Here $\phi_{0}(\vec{v})$ is the Maxwell-Boltzmann velocity distribution function, $\nu(v)$ is the collision frequency for particles with velocity $\vec{v}, \sigma(\theta)$ is the differential cross section for scattering under an angle $\theta$, and $J_{2}$ is a normalization factor obtained by replacing the logarithmic term in the numerator by 
unity and equating the resulting expression to unity. The corresponding expression for three dimensions is

$$
\begin{aligned}
h_{\mathrm{KS}} / N= & \frac{\nu}{2 \pi J_{3}} \int d \vec{v}_{1} \phi_{0}\left(\vec{v}_{1}\right) \int d \vec{v}_{2} \phi_{0}\left(\vec{v}_{2}\right)\left|\vec{v}_{12}\right| \\
& \times \int_{0}^{\pi / 2} d \theta \sin \theta \cos \theta \sigma(\pi-2 \theta) / \sigma^{2} \\
& \times \int_{0}^{2 \pi} d \phi \nu\left(v_{1}\right) \nu\left(v_{2}\right) \int_{0}^{\infty} d \tau_{1} \int_{0}^{\infty} \\
& \times d \tau_{2} e^{-\left[\tau_{1} \nu\left(v_{1}\right)+\tau_{2} \nu\left(v_{2}\right)\right]} \ln \left[2 T_{12}\left|\vec{v}_{12}\right| / \sigma\right] .
\end{aligned}
$$

To evaluate these expressions we need the velocitydependent collision frequencies for particles with velocities $\vec{v}_{1}$ and $\vec{v}_{2}$, since these frequencies are needed for the evaluation of the average of $\ln T_{12}$. For particles with interactions of finite range expressions for these collision frequencies are easily obtained from elementary kinetic theory, and explicit results can be given for $h_{\mathrm{KS}}$, after some straightforward numerical integrations. We find that for hard disks $(d=2)$

$$
h_{\mathrm{KS}} / N=\frac{\nu}{2}\left[-\ln \left(n \sigma^{2}\right)+0.209+O(n)\right]
$$

where $\nu=\left[\left(2 \pi^{1 / 2} n \sigma\right) /(\beta m)^{1 / 2}\right]$ is the average collision frequency of a dilute gas of hard disks at temperature $T$ $=\left(k_{B} \beta\right)^{-1}$, and $k_{B}$ is Boltzmann's constant. For a gas of hard spheres we find

$$
h_{\mathrm{KS}} / N=\nu\left[-\ln \left(\pi n \sigma^{3}\right)+0.562+O(n)\right],
$$

where $\nu=\left[\left(4 \pi^{1 / 2} n \sigma^{2}\right) /(\beta m)^{1 / 2}\right]$.

To check these results we have carried out extensive numerical simulations to compute the whole set of Lyapunov exponents for equilibrium hard-disk and hard-sphere systems. $h_{\mathrm{KS}}$ is obtained as the sum of all the positive exponents. In addition to the phase-space trajectory, the "exact" molecular-dynamics (MD) algorithm [12,13] follows the time evolution of a complete set of infinitesimal vectors $\delta \mathbf{X}_{l}, l=1, \ldots, 2 d N$, in tangent space by using Eqs. (6) and (7) for the streaming between collisions, and exact collision maps analogous to Eq. (9) for the discontinuous jumps at a collision. The tangent vectors are periodically reorthonormalized, and the Lyapunov exponents are obtained as the time-averaged logarithms of the renormalization factors.

The MD method requires the precise localization of the particle collision points and becomes inefficient for very low densities. We have therefore applied also a variant of the direct-simulation Monte Carlo (DSMC) technique to the computation of the Lyapunov exponents [13], where the phase-space dynamics of the system is modeled by appropriate probability distributions of the collision parameters and of the collision time, and the tangent-space dynamics remains completely deterministic [8]. For very low densities the DSMC algorithm becomes exact and is much more efficient than the MD method. It is expected to fail for high densities, for which correlated collisions become important.

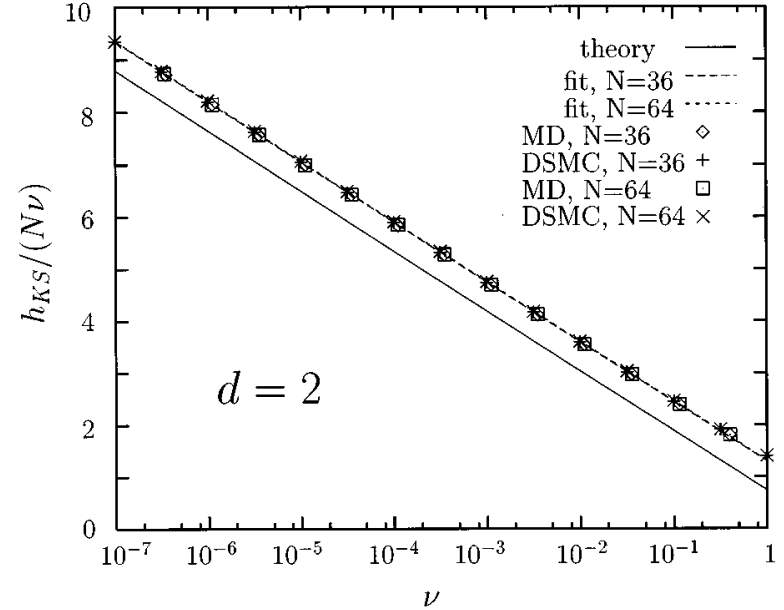

FIG. 1. $h_{\mathrm{KS}} /(N \nu)$ as a function of the collision frequency, for planar systems containing 36 and 64 hard disks. $\nu$ is measured in the reduced units introduced in the main text. The points are labeled according to the computational method MD or DSMC, the dimension $d=2$, and the number of particles $N$. The smooth line is the theoretical prediction according to Eq. (22), and the (almost undistinguishable) dashed and dotted lines are fits of Eq. (24) to the experimental points for $N=36$ and 64 , respectively. The fitting range is $10^{-7}<\nu<10^{-2}$. The fit parameters $a$ and $b$ are listed in Table I.

The simulations, performed with both methods, are for 36 and 64 disks in two dimensions, and for 32 and 108 spheres in three. The usual periodic boundary conditions are employed. To present our data we use reduced units for which the particle diameter $\sigma$, the kinetic energy per particle, $K / N=m \Sigma_{i} \vec{v}_{i}^{2} / 2 N$, and the particle mass $m$ are unity. Time is measured in units of $\left(m \sigma^{2} N / K\right)^{1 / 2}$, and the number density $n=N / V$ in units of $\sigma^{-d}$, where $V$ is the volume of the simulation box. $n$ is varied between $10^{-8}$ and 0.1 . Our choice for the unit of energy corresponds to a temperature $T$ for which $k_{B} T=K / N=1$ in two dimensions, and $k_{B} T=(2 / 3) K / N$ $=2 / 3$ in three. The dependence of $h_{\mathrm{KS}} /(N \nu)$ on the collision frequency $\nu$ is displayed in Figs. 1 and 2 for the respective two- and three-dimensional systems studied here. As indicated by the labels, the diamonds and squares denote MD results, whereas the plus signs and crosses refer to DSMC data. It is obvious from the figures, that there is very good agreement between the MD and DSMC results for $h_{\mathrm{KS}} / N \nu$, and that this agreement persists even to the largest collision frequencies of interest. We therefore do not distinguish in the following discussion between these two independent sets of simulation data.

The particle-number dependence of the KolmogorovSinai entropy per particle is found to be very small for hard disks, with the points for 36 and 64 particles practically undistinguishable on the scale of Fig. 1. For hard spheres there is a noticeable difference between the results for $N=32$ (dashed) and 108 (dotted) in Fig. 2. However, it is expected that the larger of the two systems is already close to the thermodynamic limit, and that a comparison of our finiteparticle data with the theoretical prediction, valid for $N \rightarrow \infty$, is meaningful.

To facilitate this comparison, we rewrite Eqs. (22) and (23) according to 


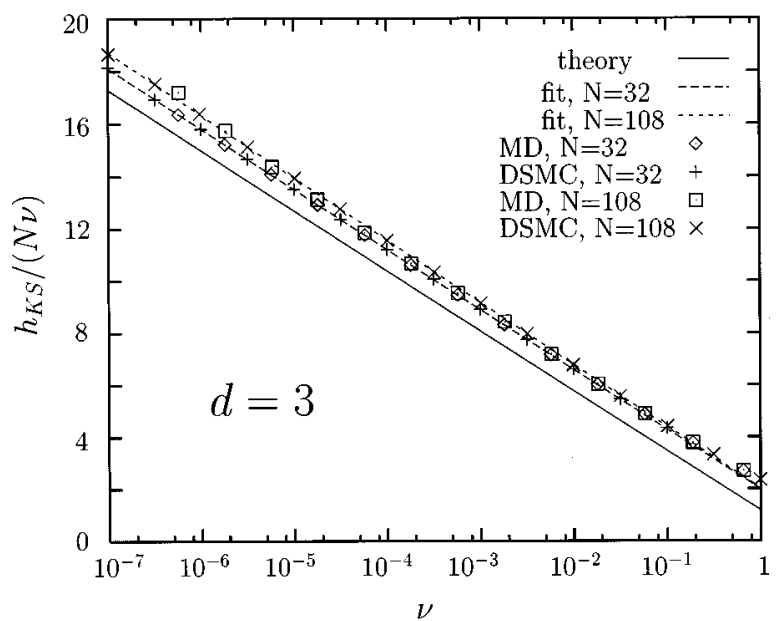

FIG. 2. $h_{\mathrm{KS}} /(N \nu)$, as a function of the collision frequency, for three-dimensional systems containing 32 and 108 hard spheres. $\nu$ is measured in the reduced units introduced in the main text. The points are labeled according to the computational method, MD or DSMC, the dimension, $d=3$, and the number of particles $N$. The smooth line is the theoretical prediction according to Eq. (23), and the dashed and dotted lines are fits of Eq. (24) to the experimental points for $N=32$ and 108 , respectively. The fitting range is $10^{-7}$ $<\nu<10^{-2}$. The fit parameters $a$ and $b$ are listed in Table I.

$$
h_{\mathrm{KS}} /(N \nu)=a\left[-\ln \left(\nu / \nu_{0}\right)+b\right]+O\left(\nu^{2}\right),
$$

where $\nu_{0}=2\left[\pi /\left(m \sigma^{2}\right)\right]^{1 / 2}(K / N)^{1 / 2}$ for hard disks, and $\nu_{0}$ $=4\left(\pi m \sigma^{2}\right)^{-1 / 2}(2 K / 3 N)^{1 / 2}$ for hard spheres. Neglecting the higher-order terms and treating $a$ and $b$ as fit parameters, this expression is fitted to the experimental data for $h_{\mathrm{KS}} /(N \nu)$ in the range $10^{-7}<\nu<10^{-2}$. The results for $a$ and $b$ are summarized in Table I. The theoretical expectation for $h_{\mathrm{KS}} /(N \nu)$ is indicated by the smooth line in Fig. 1, $\{a, b\}$ $=\{0.5,0.209\}$, for disks, and in Fig. $2,\{a, b\}=\{1,0.562\}$, for spheres.

TABLE I. Fit parameters for the fit of Eq. (23) to the experimental $h_{\mathrm{KS}} /(N \nu)$, for a system containing $N$ hard disks $(d=2)$, or spheres $(d=3)$. The fitting range is $10^{-7}<\nu<10^{-2}$, where $\nu$ is the collision frequency in the reduced units introduced in the main text. MD and DSMC refer to the numerical method used for the computation. The quoted errors for the fit parameters $a$ and $b$ are standard deviations.

\begin{tabular}{ccccc}
\hline \hline$d$ & $N$ & Method & $a$ & $b$ \\
\hline 2 & 36 & MD & $0.499 \pm 0.001$ & $1.339 \pm 0.003$ \\
2 & 36 & DSMC & $0.500 \pm 0.001$ & $1.326 \pm 0.006$ \\
2 & 64 & MD & $0.499 \pm 0.001$ & $1.366 \pm 0.005$ \\
2 & 64 & DSMC & $0.500 \pm 0.001$ & $1.358 \pm 0.009$ \\
2 & $\infty$ & Theory & 0.5 & 0.209 \\
3 & 32 & MD & $0.998 \pm 0.001$ & $1.42 \pm 0.01$ \\
3 & 32 & DSMC & $1.000 \pm 0.001$ & $1.38 \pm 0.01$ \\
3 & 108 & MD & $1.03 \pm 0.03$ & $1.34 \pm 0.29$ \\
3 & 108 & DSMC & $1.036 \pm 0.002$ & $1.34 \pm 0.02$ \\
3 & $\infty$ & Theory & 1.0 & 0.562 \\
\hline \hline
\end{tabular}

From an inspection of Table I we conclude that the theoretically expected value for $a$, which determines the slope of the straight lines in the figures, is almost perfectly reproduced by the fits. The theoretical values for the other parameter $b$, however, are not recovered by the fitting procedure. This is reflected in the offset of the theoretically expected smooth line with respect to the experimental results in the Figs. 1 and 2.

To shed some light on the origin of this disagreement, we numerically evaluated Eq. (16) by averaging over a molecular-dynamics simulation run. We find perfect agreement of this average with the theoretical predictions of Eqs. (22) and (23). Moreover, a computer simulation where the terms $\delta \vec{r}_{k, 0}$ and $\delta \vec{r}_{k, 0}$ are set equal to zero for each $k-l$ collision yields results that do agree with Eqs. (22) and (23), but not with the results where these spatial displacements are taken into account. Thus, there appears to be a problem with the neglect of the spatial deviations $\delta \vec{r}_{l, 0}-\delta \vec{r}_{k, 0}$ in going from Eq. (11) to Eq. (14). That is, the neglect of $\delta \vec{r}_{l, 0}$ $-\delta \vec{r}_{k, 0}$ in Eq. (11) has not yet been justified by an analytic calculation where such terms are included. In fact, a more detailed analysis reveals that indeed the inclusion of the neglected terms yields corrections to the coefficient $b$, which may crudely be estimated to be close to $\ln 2$. However, these are very preliminary results which must be verified by more extensive calculations. It is worth noting that in the case of the two- and three-dimensional Lorentz gas the neglect of spatial deviations in the equivalent of Eq. (14) can be fully justified, and that there the theoretical results for the analogs of the coefficients $a$ and $b$ agree very well with the computed simulations $[5-8,14]$. Finally notice that the assumptions made do not affect the theoretical values of the leading term involving only the parameter $a$. Presently we are working both on calculations of the terms neglected above and on a more systematic approach using methods based upon the Bogoliubov-Born-Green-Kirkwood-Yuon hierarchy equations, which will provide a systematic density expansion of $h_{\mathrm{KS}}$.

In conclusion, we have obtained the first theoretical values for the leading term in the KS entropy per particle for dilute gases with short-range forces, both for two- and threedimensional systems. The comparison of these predictions with results of computer simulations are excellent, but we do not yet have a final analytic expression for the first-order corrections to these results. The computer simulations, however, do provide accurate values for these corrections, to which analytic results can eventually be compared. There seems to be little doubt that our results can be extended to dense gases and to nonequilibrium states of interest for the escape-rate or the Gaussian thermostat approaches for relating transport coefficients to the chaotic properties of fluid systems.

The authors are pleased to acknowledge the hospitality of the Erwin Schrödinger Institut of the Universität Wien where this work was initiated. We would like to thank Dr. Arnulf Latz and Ramses van Zon for helpful comments on this and related subjects; Dr. Charles Ferguson for helpful comments and assistance with numerical integrations; and the National 
Science Foundation for support under Grant No. NSF-PHY96-00428. H. A. P. and C. D. gratefully acknowledge support from the Fonds zur Förderung der wissenschaftlichen Forschung, under Grant Nos. P09677-PHY, P11428-PHY, and
J01302-PHY, and H. vB. acknowledges support by FOM, SMC, and by the NWO Priority Program Non-Linear Systems, which are financially supported by the "Nederlandse Organisatie voor Wetenschappelijk Onderzoek (NWO)."
[1] E. Ott, Chaos in Dynamical Systems (Cambridge University Press, Cambridge, 1993).

[2] J.-P. Eckmann and D. Ruelle, Rev. Mod. Phys. 57, 617 (1985).

[3] P. Gaspard and G. Nicolis, Phys. Rev. Lett. 65, 1693 (1990).

[4] J. R. Dorfman and P. Gaspard, Phys. Rev. E 51, 28 (1995); P. Gaspard and J. R. Dorfman, ibid. 52, 3525 (1995).

[5] H. van Beijeren and J. R. Dorfman, Phys. Rev. Lett. 74, 4412 (1995); 76, 3238(E) (1996); Physica A 240, 12 (1997).

[6] H. van Beijeren, J. R. Dorfman, E. G. D. Cohen, H. A. Posch, and Ch. Dellago, Phys. Rev. Lett. 77, 1974 (1996).

[7] A. Latz, H. van Beijeren, and J. R. Dorfman, Phys. Rev. Lett.
78, 207 (1997); (unpublished).

[8] Ch. Dellago and H. A. Posch, Phys. Rev. Lett. 78, 211 (1997).

[9] M. H. Ernst, J. R. Dorfman, R. Nix, and D. Jacobs, Phys. Rev. Lett. 74, 4416 (1995).

[10] C. Appert, H. van Beijeren, M. H. Ernst, and J. R. Dorfman, Phys. Rev. E 54, R1013 (1996); J. Stat. Phys. 87, 1253 (1997).

[11] H. van Beijeren, and R. van Zon (unpublished).

[12] Ch. Dellago, H. A. Posch, and W. G. Hoover, Phys. Rev. E 53, 1485 (1996).

[13] Ch. Dellago and H. A. Posch, Physica A 240, 68 (1997).

[14] Ch. Dellago and H. A. Posch, Phys. Rev. E 52, 2401 (1995). 